Due to the COVID-19 pandemic, many 2020 events have been canceled or have moved to a virtual platform. Please check the event website for additional information concerning each listing.

\footnotetext{
List your meeting here:

Following is a list of microscopy related meetings and courses. The editors would greatly appreciate input to this list via the electronic submission form under "events/other microscopy events" at the MSA webpage: www.microscopy.org. We will gladly add hypertext links to the notice on the web and insert a listing of the meeting in the next issue. Please furnish the following information (any additional information provided will be edited as required and printed on a space available basis):

- Meeting/Course name

- Meeting/Course dates

- Meeting/Course topic or short description

- Sponsoring society/organization/university

- Contact person

- Telephone number

- Fax number

- Email address

- URL for website
}

\section{Meetings 2020}

\section{ASM Annual Meeting (IMAT): Solving \\ Global Materials Challenges \\ September 14-17, 2020 \\ Cleveland, $\mathrm{OH}$ \\ www.asminternational.org/web/imat}

\section{6th Annual NSH Symposium/ Convention}

October 13-15, 2020

Virtual meeting

Sponsor: National Society for Histotechnology www.histoconvention.org/index.cfm

\section{Neuroscience 2020}

October 24-28, 2020

Washington, DC

Sponsor: Society for Neuroscience

www.sfn.org/meetings/neuroscience-2020

\section{MRS Fall Meeting \& Exhibit}

November 29-December 4, 2020

Boston, MA

www.mrs.org/fall2020
ASCB 2020 Annual Meeting

Virtual meeting, dates TBD

(previously December 5-9, 2020)

www.ascb.org/cellbio2020

\section{Courses 2020}

\section{Exchange of Experience V}

September 7, 2020

Okazaki, Japan

www.globalbioimaging.org/exchange-ofexperience

Introduction to Microscopy Techniques

September 15-17, 2020

Hatfield, PA

Sponsor: Electron Microscopy Sciences www.emsmicroscopyacademy.com/productpage/intro-sep20

\section{Materials Ultramicrotomy}

September 21, 2020 (Beginner)

September 22-24, 2020 (Some Experience)

Hatfield, PA

Sponsor: Electron Microscopy Sciences www.emsmicroscopyacademy.com/productpage/materials-sep20

\section{Sample Preparation Techniques}

September 29-October 1, 2020

Westmont, IL

Sponsor: Hooke College of Applied Sciences www.mccrone.com/courses/samplepreparation-techniques

\section{Cryo SEM}

October 13-15, 2020

Hatfield, PA

Sponsor: Electron Microscopy Sciences www.emsmicroscopyacademy.com/productpage/cryosem-oct20

\section{Biological SEM}

October 20-22, 2020

Hatfield, PA

Sponsor: Electron Microscopy Sciences www.emsmicroscopyacademy.com/productpage/biosem-oct20

\section{Scanning Electron Microscopy:} Advanced X-ray Microanalysis by EDS November 9-11, 2020

Westmont, IL

Sponsor: Hooke College of Applied Sciences www.mccrone.com/courses/sem-advanced-eds
Aurion Immunogold Silver Staining

November 10-12, 2020

Hatfield, PA

Sponsor: Electron Microscopy Sciences www.emsmicroscopyacademy.com/productpage/immunogold-nov20

\section{Scanning Electron Microscopy:}

Advanced Imaging Practices

November 12-13, 2020

Westmont, IL

Sponsor: Hooke College of Applied Sciences www.mccrone.com/courses/sem-advancedimaging

\section{Biological TEM}

November 17-19, 2020

Hatfield, PA

Sponsor: Electron Microscopy Sciences www.emsmicroscopyacademy.com/productpage/biotem-nov20

\section{Meetings 2021}

\section{ABRF 2021}

March 7-10, 2021

Hynes Convention Center, Boston, MA

https://abrf.org/meetings

\section{$5^{\text {th }}$ International Conference on BioTribology}

April 25-29, 2021

Banff, Alberta, Canada

www.elsevier.com/events/conferences/ international-conference-on-biotribology

\section{EMAS 2021 - 17 th European Workshop} on Modern Developments and Applications in Microbeam Analysis

May 16-20, 2021

Krakow, Poland

www.microbeamanalysis.eu/events/ event/60-emas-2021-17th-europeanworkshop-on-modern-developments-andapplications-in-microbeam-analysis

$23^{\text {rd }}$ Biennial Meeting of the International Society of Developmental Neuroscience

\section{May 22-25, 2021}

Vancouver, Canada

www.isdn-conference.elsevier.com 
$15^{\text {th }}$ International Conference on Macromolecular Chemistry and Molecule Microscopy - ICMCMM 2021 June 24-25, 2021

Oslo, Norway

https://waset.org/macromolecular-chemistryand-molecule-microscopy-conference-injune-2021-in-oslo

mmc2021: Microscience

Microscopy Congress 2021

July 5-8, 2021

Manchester, UK

www.mmc-series.org.uk

Microscopy \& Microanalysis 2021

August 1-5, 2021

Pittsburgh, PA

Email:nicoleguy@conferencemanagers.com www.microscopy.org

\section{Microscopy Conference}

(Dreiländertagung) MC 2021

August 22-26, 2021

Vienna, Austria

www.microscopy-conference.de

$16^{\text {th }}$ International Congress

of Histochemistry and

Cytochemistry (ICHC)

September 5-8, 2021

Prague, Czech Republic

http://ichc2020.com

$3^{\text {rd }}$ International Conference On

Nanoscopy - ICON EUROPE 2021

September 6-9, 2021

Jena, Germany

www.icon-europe.org

The $9^{\text {th }}$ Electron Tomography Congress September 12-15, 2021

Egmond aan Zee, The Netherlands

https://tomo2020.org

\section{$4^{\text {th }}$ Annual NSH Symposium/ \\ Convention}

September 16-23, 2021

Omaha, NE

Sponsor: National Society for Histotechnology www.histoconvention.org/futuredates.cfm

$9^{\text {th }}$ International Conference

on Fracture of Polymers,

Composites and Adhesives

September 26-30, 2021

Les Diablerets, Switzerland

www.elsevier.com/events/conferences/

esistc4conference

Neuroscience 2021

November 13-17, 2021

Chicago, IL

Sponsor: Society for Neuroscience

www.sfn.org
2021 MRS Fall Meeting

November 28-December 3, 2021

Boston, MA

www.mrs.org/fall2021

\section{ASCB 2021 Annual Meeting}

December 11-15, 2021

San Diego, CA

www.ascb.org/meetings-events/future-ascbmeetings

\section{Courses 2021}

GWNIC Correlative Light \&

Electron Microscopy Workshop

June 2021, exact dates TBD

Washington, DC

https://nic.gwu.edu/clem-workshop

\section{Meetings 2022}

\section{ABRF 2022}

March 27-30, 2022

Palm Springs Convention Center, Palm Springs, CA https://abrf.org/meetings

\section{FENS Forum 2022}

July 9-13, 2022

Paris, France

Email: forum2022@fens.org

www.fens.org/News-Activities/Calendar/

Meetings/2022/07/FENS-Forum-2022

Microscopy \& Microanalysis 2022

July 31-August 4, 2022

Portland, OR

Email: nicoleguy@conferencemanagers.com www.microscopy.org

IMC20: 20th International Microscopy

Congress

September 25-30, 2022

Busan, Korea

https://uia.org/other-news/30413

\section{Meetings 2023}

Microscopy \& Microanalysis 2023

July 24-28, 2023

Minneapolis, $\mathrm{MN}$

www.microscopy.org

\section{Meetings 2024}

Microscopy \& Microanalysis 2024

July 28-August 1, 2024

Cleveland, $\mathrm{OH}$

www.microscopy.org
Expand your Knowledge

of Microscopy with

MSA
Membership!

Whether your primary focus is in light, electron, or scanning probe microscopy, or the biological or physical sciences, MSA takes your knowledge to the next level!

\section{Members Receive:}

- A personal subscription to MSA's official journal, Microscopy and Microanalysis, and MSA's popular bi-monthly magazine, Microscopy Today

- Peer Networking through the Society's Focused Interest Groups and Local Affiliated Societies

- Discounts on books, journals, and other educational materials

- MSA Awards Programs, Speaker Opportunities, Scholarships, and much more!

For more information: visit www.microscopy.org

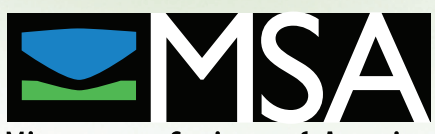

Microscopy Society of America Join MSA Today! 